\title{
Determining an optimum zone radius for zone routing protocol (ZRP) based on node mobility
}

\author{
Khalid W. Al-Ani ${ }^{1}$, Salman Yussof ${ }^{2}$, Hussein M. Haglan ${ }^{3}$, Hothefa Shaker ${ }^{4}$, Linda Mahdi Alani $^{5}$ \\ ${ }^{1,3}$ College of Information Technology, Universiti Tenaga Nasional, Selangor, Malaysia \\ ${ }^{1,3}$ Presidency University, University of Anbar, Ramadi, Anbar, Iraq \\ ${ }^{2}$ Institute of Informatics and Computing in Energy, Universiti Tenaga National \\ ${ }^{4}$ Modern College of Business and Science, Oman \\ ${ }^{5}$ Divan of Sunni Endowment, Department of Religious Education and Islamic Studies, Baghdad, Iraq
}

\section{Article Info \\ Article history: \\ Received Jul 26, 2020 \\ Revised Sep 27, 2020 \\ Accepted Oct 13, 2020}

\section{Keywords:}

Hybrid routing

MANET

Proactive routing

Reactive routing

ZRP

\begin{abstract}
Mobility is one of the important issues in mobile ad hoc networks (MANET). By definition, MANET nodes are free to move around and this may cause difficulty in routing. MANET routing protocols must consider this factor when making routing decision. zone routing protocol (ZRP) is a hybrid routing protocol, which utilizes the proactive and reactive routing protocols advantages. ZRP proactively maintains routing information within a routing zone, while reactively discovering routes to destinations beyond the routing zone. Since ZRP is based on the concept of routing zone, determining an optimum routing zone radius has the major impact on the performance of that protocol. In this research, we studied the effect of zone radius on the performance of ZRP with different levels of node mobility. Node mobility is defined using two different parameters: node speed and pause time. Based on the simulation results, ZRP protocol using zone radius of two provides the best packet delivery fraction, throughput and normalized routing load. However, a larger zone radius will provide a lower delay.
\end{abstract}

This is an open access article under the CC BY-SA license.

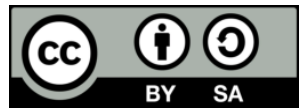

\section{Corresponding Author:}

Khalid W. Al-Ani

Department of Studies and Planning

University of Anbar, Anbar, Iraq

Email: khaled_alani@uoanbar.edu.iq

\section{INTRODUCTION}

MANET is a type of wireless network that does not require infrastructure or stations to connect to a group of mobile nodes. The need for such networks appeared to establish communications in emergency circumstances in which the infrastructure was not available [1-3]. MANET networks can be used in many applications such as building virtual classrooms, in military wars, natural disasters such as earthquakes and floods, and other applications [4-7]. The length of the communication path between two people using the MANET network consists of one-hop or many hops. Each device within the network is called a node, and these nodes work to receive and send messages and as a router at the same time, they direct messages to other nodes in order to maintain the continuity of the connection $[8,9]$. Each node within these networks moves freely and randomly within the network, and it may exist from the network at any time [10, 11]. Due to the constant and random movement of nodes in and out of the network, the MANET network topology is always variable $[12,13]$. Therefore, these networks used many routing protocols and there is still a great need to find ideal protocols to establish communication within these networks [14-16]. 
Proactive, Reactive, and hybrid are the three main types of routing protocols in MANET networks $[17,18]$. One of the advantages of proactive-routing protocols is the speed of sending packets from the source to the target, because it has saved the network topology through saving the route on each node in the network in a special table called the routing table and it works on updating it always [19]. One of the advantages of reactive-routing protocols is the low load because they do not overload the network with control messages that were used to update the previous type, but rather send control messages only upon request, providing a route to send a packet from a source to a specific target [20]. The advantages of proactive and reactive routing protocols have been exploited to build the third type called hybrid-routing protocols, which use proactive-protocol techniques to deliver packets to nodes within the network while using reactive-protocol techniques to deliver packets to nodes outside the network [21, 22].

The limitations of excess control overhead and delay of long route requests for proactive and reactive routing protocols are addressed in the design of ZRP by combining the advantages of these paradigms [23]. ZRP utilizes the proactive paradigm inside the zone and reactive paradigm outside the zone. A routing zone of a node represents the area of the local neighborhood of that node, and each node could be part of multiple overlapping zones. The size of the zone is determined by the radius, the length of the radius is a number of hops from a specific node to the boundaries of the zone [24].

The zone radius is considered one of the most important factors for the performance of ZRP because it works to balance the performance of the network by reducing the load and delay by dividing the network area into zones. The routing process in these areas is managed by two techniques of proactive and reactive routing. Proactive-routing is used inside the zone and reactive-routing outside the zone. Decreasing the radius value will reduce the area of proactive-routing and increase the area of reactive-routing, thus reduce the load on the network and increase the delay in the messages routing process, and vice versa when increasing the radius value.

The node's mobility is very important and it has a great impact on the performance of the network and depends mainly on speed and pause factors. When increasing the speed of the nodes and reducing the pause time, the network topology will constantly change because the nodes will constantly change their locations in and out of the network and this will increase the load on the network and increase the loss of communication with the nodes due to the nodes departure or consumption of their energy. But the opposite happens when the node speed is reduced and the pause is increased, the network topology will become more stable, the chances of communication between the nodes will increase, thus reducing the routing load on the network and reducing energy consumption.

There is not much research on hybrid routing protocols. On the contrary, most of the research in previous years has given a lot of research effort on proactive and reactive routing protocols to provide communication between nodes in MANET networks. Optimized Link State Routing (OLSR) [25] is an example of a proactive-routing protocol, Dynamic Source Routing (DSR) [26] and Ad Hoc On-Demand Distance Vector Routing (AODV) [27] are examples of reactive-routing protocols. Therefore in this paper, we will work on study one of the most used hybrid routing protocols called zone routing protocol (ZRP), and try to find out the effect of the zone radius on the performance of ZRP by using a different number of zone radius in several cases for ZRP to determine the best zone radius that can be used in ZRP. Two different network environments were used to apply these cases. First environment, when the speed of the nodes is variable. The second environment, when pause time is variable.

\section{SIMULATION SETUP}

A simulation model for the ZRP protocol was built using the most famous simulation tool called the Network Simulator NS2 version 2.33 (which is one of the most popular open-source network simulators [28]) by preparing two different scenarios. In the first scenario: the network was simulated through four cases of ZRP. In each case, the radius of the zone varied from one case to another, where it was increased gradually from $2,3,4$, to 5 hops from the first case to the fourth case respectively. 50 nodes were used in the network area. Each node had: a transmission range of 50 meters, a pause time was changed from 0 (the highest movement) to 100 seconds (no movement), while the node speed of the node was 20 meters/second. These nodes move randomly in an environment from 1500 meters * 1500 meters, and the simulation time is set to 900 seconds.

In scenario 2, 50 nodes are distributed within $1500 \times 1500$ area and the transmission range is $250 \mathrm{~m}$ while the simulation period is set to 900 seconds. The movement of the nodes was at a speed between 20-100 $\mathrm{ms}$ and different routing zone were used from 2 to 5 while the pause time is fixed to 20 seconds. Table 1 shows the parameters for scenarios 1 and 2. 
Table 1. Simulation parameters for two scenarios

\begin{tabular}{ccc}
\hline Simulation parameters & Scenario 1 & Scenario 2 \\
\hline Number of Nodes & 50 nodes & 50 nodes \\
Simulation Time & 900 seconds & 900 seconds \\
Map Size & $1500 \mathrm{~m} \mathrm{x} 1500 \mathrm{~m}$ & $750 \mathrm{~m} \mathrm{x} 750 \mathrm{~m}$ \\
Max Speed & $20 \mathrm{~m} / \mathrm{s}$ & $20,40,60,80$ and $100 \mathrm{~m} / \mathrm{s}$ \\
Mobility Model & Random waypoint & Random waypoint \\
Traffic Type & Constant bit rate $(\mathrm{CBR})$ & Constant bit rate $(\mathrm{CBR})$ \\
Packet Size & 512 bytes & 512 bytes \\
Connection Rate (Nominal Radio Range) & 4 pkts $/ \mathrm{sec}$ & $4 \mathrm{pkts} / \mathrm{sec}$ \\
Pause Time & $0,20,40,60,80,100$ seconds & 20 second \\
Number of Connection & 5 & 5 \\
Bandwidth of links & $2 \mathrm{Mbit}$ & $2 \mathrm{Mbit}$ \\
MAC layer type & IEEE 802.11 & IEEE 802.11 \\
\hline
\end{tabular}

\section{PERFORMANCE METRICS}

Performance metrics are used to measure a routing protocol to identify the effective and best route to the destination. There are several performance metrics such as throughput (TP), "Packet Delivery Fraction (PDF)", "Normalized Routing Load (NRL)", "average end-to-end delay (E-to-E delay)", "Total Packet Received", "Data Packet Dropped", and some other metrics. In this simulation, the performance of the routing protocol will be evaluated based on the following four performance metrics:

a) Packet Delivery Fraction (PDF): It is the data packets received by destination per data packets send by sources [29].

$$
\text { Packet Delivery Fraction }=\frac{\sum \text { "packets received by destinations" }}{\sum \text { "packets sent by sources" }} \times 100
$$

b) Throughput (TP): To measure the efficiency of the protocol's performance in terms of routing and receiving data packets that are actually arriving at their destinations by finding the average percentage of data packets that have succeeded in reaching their destinations during the simulation time. The throughput is calculated by the following equation [30]:

$$
\text { Throughput }=\frac{\sum \text { "packets received by destinations" }}{\text { simulation time }}
$$

c) Average E-to-E delay: Used to measure the average time spent by packets on their journey from the sending nodes to the receiving nodes [18].

$$
\text { Average } \mathrm{E}-\text { to }-\mathrm{E} \text { delay }=\frac{\sum \text { "packet received time-packet sent time" }}{\sum \text { "packets received by destinations" }}
$$

d) Normalized Routing Load (NRL): It is used to calculate the ratio between the numbers of routing messages that travel through the network to the number of actual data packets reaching their targets. The most important of NRL is to measure the scalability of the routing protocol and its efficiency related to consuming node battery power [24].

$$
\text { Normalized Routing Load (NRL) }=\frac{\sum \text { "Transmitted Routing Packets" }}{\sum \text { "Packets received by the destinations" }}
$$

\section{SIMULATION RESULTS AND ANALYSIS}

In this simulation, four kinds of performance metrics such as "Throughput", "Normalized Routing Load (NRL)", "average end-to-end delay", and "Packet delivery Fraction (PDF)" are used in the performance comparisons and evaluations. The results that have been obtained from the simulation of ZRP had been overviewed and analyzed depending on the performance measurements as shown in the following sections.

\subsection{Results and analysis of PDF}

Figures 1 and 2 shows the simulation results for ZRP in the two scenarios in terms of PDF In Figure 1 , it is noted that with the increase of pause time, all the four test cases show an increase in packet delivery fraction. ZRP with zone radius 2 increases from $58.02 \%$ to $76.57 \%$, ZRP with zone radius 3 increases from 
$53.54 \%$ to $72.46 \%$, ZRP with zone radius 4 increases from $49.24 \%$ to $65.31 \%$, and ZRP with zone radius 5 increases from $45.25 \%$ to $61.51 \%$ respectively when the pause time increases. The reason that is the nodes stop moving due to the increase in pause time. Thus, the probability of a broken link is lower and the network becomes stable. The increment of PDF in ZRP with zone radius 2 is $31.97 \%, 35.34$ for ZRP with zone radius 3, 32.64 for ZRP with zone radius 4, 35.93 for ZRP with zone radius 5. The increase in PDF of the four test cases is slightly different. However, ZRP with zone radius 2 shows better PDF as compared with the three test cases. In zone radius 2, most of the time ZRP will behave like a purely reactive routing to find the route to the destination by using route discovery mechanism and the overhead related to the number of Inter-zone Routing Protocol (IERP) packets is relatively small as compared with the number of Intra-zone Routing Protocol (IARP) packets since it is generated upon request. Hence, the PDF is increased.

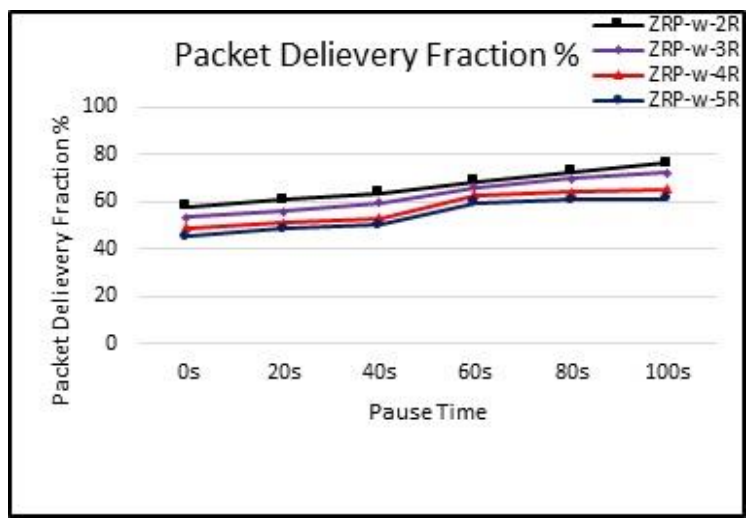

Figure 1. PDF for scenario 1

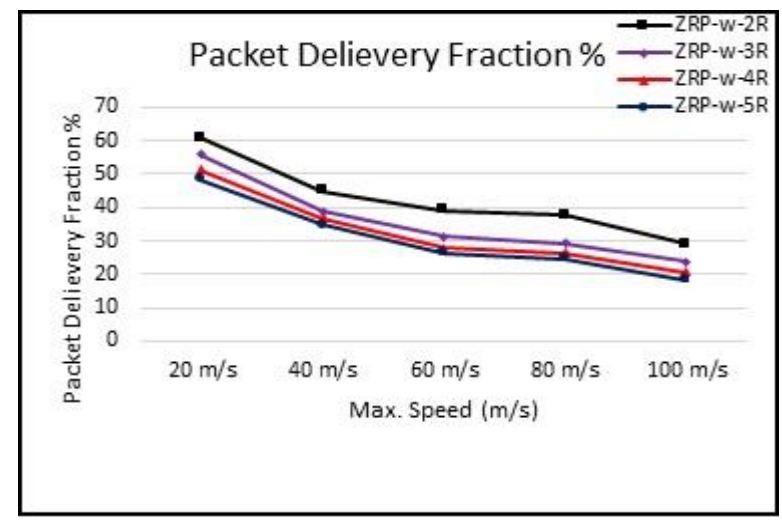

Figure 2. PDF for scenario 2

In Figure 2, when the maximum speed of nodes increases from 20 to $100 \mathrm{~m} / \mathrm{s}$, all the four test cases show a decrease in PDF. The PDF for ZRP with zone radius 2 decreases from $60.87 \%$ to $28.94 \%$, ZRP with zone radius 3 decreases from $55.85 \%$ to $23.81 \%$, ZRP with zone radius 4 decreases from $51.23 \%$ to $20.54 \%$, ZRP with zone radius 5 decreases from $48.52 \%$ to 18.31 . The increase in node speed causes links between nodes are easily broken and this will lead to lower PDF. It is also observed that the decrease in PDF for the four-zone radius is very similar. However, the percentage of decrease of the ZRP with zone radius 2 is lower as compared to another zone radius, where there is a $52.46 \%$ decrease in PDF as compared to $57.37 \%$ for ZRP with zone radius 3, 59.91\% for ZRP with zone radius 4, 62.26\% for ZRP with zone radius 5. The reason is that with a small zone radius, most of the time ZRP will behave like a reactive routing protocol, i.e. IERP in which there is more than one route reply (RREP) for every route request (RREQ) and the track of more than one path between source and destination is kept by ZRP. In the case where one path is broken, an alternative path is available and chosen; hence, the decrease of PDF for ZRP with zone radius 2 is lower than ZRP with zone radius 3, 4, and 5 respectively.

\subsection{Results and analysis of average E-to-E Delay}

Figures 3 and 4 show the simulation results for ZRP in the two scenarios in terms of average E-to-E delay. In Figure 3, the average E-to-E delay decreases for all the four test cases when the pause time increases. The average E-to-E delay of zone radius 2 decreases from 570.012 to $435.49 \mathrm{~ms}$, ZRP with zone radius 3 decreases from 562.59 to $402.75 \mathrm{~ms}$, ZRP with zone radius 4 decreases from 546.875 to $335.731 \mathrm{~ms}$, ZRP with zone radius 5 decreases from 536.387 to $290.259 \mathrm{~ms}$ The percentage of decrease of zone radius 2 is $23.60 \%, 28.41 \%$ for ZRP with zone radius 3, 38.61\% for ZRP with zone radius 4, 45.89\% for ZRP with zone radius 5 . This is because when the pause time increases, the node movement decreases, and the network becomes more stable. Thus, broken or loss of the path that usually occurs due to the higher movement of the nodes was less and this results in lower average E-to-E delay. It is also observed that there is no effect on the performance of ZRP by zone radius in terms of average E-to-E delay when the pause time is set to 0s. ZRP with zone radius 5 shows a lower average E-to-E delay compared to ZRP with zone radius 2, 3, and 4. The reason is that when the zone radius increases, the scope of a proactive routing mechanism (IARP) also increases. IARP has the lowest latency due to its ability to maintain the routing information up-to-date. Hence, the average E-to-E delay decreases. 


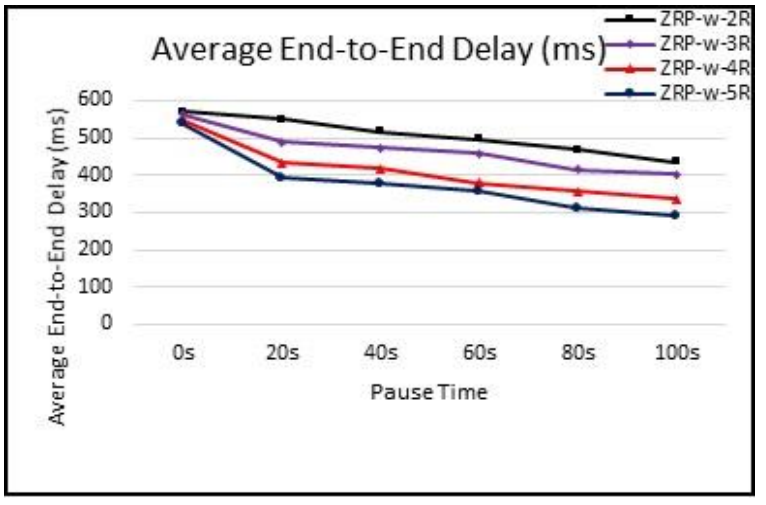

Figure 3. Average E-to-E delay for scenario 1

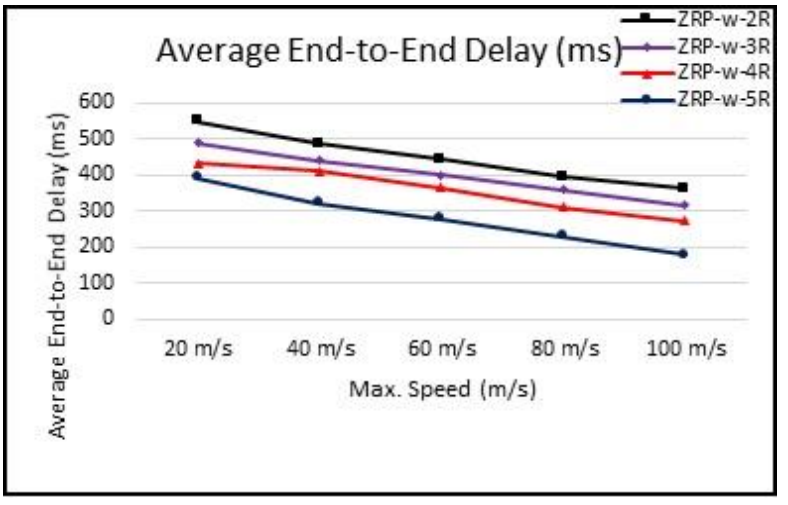

Figure 4. Average E-to-E delay for scenario 2

In Figure 4, when maximum node speed increases from 20 to $100 \mathrm{~m} / \mathrm{s}$, the average E-to-E delay decreases for all of the four test cases. The average E-to-E delay of ZRP with zone radius 2 decreases from 549.496 to $363.12 \mathrm{~ms}$, ZRP with zone radius 3 decreases from 488.893 to $315.578 \mathrm{~ms}$, ZRP with zone radius 4 decreases from 433.121 to $273.167 \mathrm{~ms}$, and ZRP with zone radius 5 decreases from 393.32 to $178.141 \mathrm{~ms}$. The reason is that since ZRP is a hybrid routing protocol, it provides a balance between overhead caused by proactive routing and latency caused by reactive routing. Therefore, when the node speed increases, the E-toE delay of all the four test cases decreases as well. The percentage of decrease of ZRP with zone radius 2 is $33.92 \%, 35.45 \%$ for ZRP with zone radius 3, 58.55\% for ZRP with zone radius 4, and 54.71 for ZRP with zone radius 5. Figure 4 also shows that ZRP with zone radius 5 has lower E-to-E delay compared to ZRP with zone radius 2,3 , and 4 . The reason is that with a large zone radius, the scope of a proactive routing mechanism (IARP) will be increased to keep the routing information up-to-date of a zone. In the case of link failure due to node movement, an alternative path is already available and can be immediately used, hence decreasing average E-to-E delay.

\subsection{Results and Analysis of TP}

Figures 5 and 6 show the simulation results for ZRP in the two scenarios in terms of TP. In Figure 5, it is noted that with the increase of pause time, all the four test cases show an increase in TP. ZRP with zone radius 2 increases from $11.2 \mathrm{kbps}$ to $14.78 \mathrm{kbps}$, ZRP with zone radius 3 increases from $10.24 \mathrm{kbps}$ to 13.94 $\mathrm{kbps}$, ZRP with zone radius 4 increases from $9.41 \mathrm{kbps}$ to $12.56 \mathrm{kbps}$, ZRP with zone radius 5 increases from $8.69 \mathrm{kbps}$ to $11.78 \mathrm{kbps}$ respectively when the pause time is increased from 0 to 100 seconds. This is because the lower node movement leads to a lower possibility of the link failure and causes the network to become more stable. As a result, network TP increases. It is also observed that all four test cases have a very similar increase in TP. The percentage of TP increase of ZRP with zone radius 2 is $31.96 \%, 36.13 \%$ for ZRP with zone radius 3, 33.48\% for ZRP with zone radius 4 , and $35.56 \%$ for ZRP with zone radius 5. ZRP with zone radius 2 has a higher TP compared to the other three test cases. The reason is that with a small zone radius, most of the time ZRP will behave like a reactive routing mechanism (IERP) in which the Bordercast Resolution Protocol (BRP) is used to send route queries to the peripheral nodes and directing route requests away from the covered routing zone using query control mechanisms. As a result, the routing load is reduced and redundant queries are also minimized. Hence, ZRP with a small zone radius gets high TP.

Figure 6 shows that with increasing node speed, all the four test cases show a decrease in TP when the maximum speed of nodes increases from 20 to $100 \mathrm{~m} / \mathrm{s}$. The TP of ZRP with zone radius 2 decreases from $11.87 \mathrm{kbps}$ to $5.6 \mathrm{kbps}$, ZRP with zone radius 3 decreases from $10.72 \mathrm{kbps}$ to $4.55 \mathrm{kbps}$, ZRP with zone radius 4 decreases from $10.18 \mathrm{kbps}$ to $3.76 \mathrm{kbps}$, and ZRP with zone radius 5 decreases from $9.49 \mathrm{kbps}$ to $3.53 \mathrm{kbps}$ respectively. High node speed leads to topology change of the network and the probability of link failure increases. Therefore, the network TP decreases as node speed increases. ZRP with zone radius 2 shows a higher level of robustness against network topology change where the percentage of decrease in TP is only $52.82 \%$, as compared to $57.55 \%$ for ZRP with zone radius $3,63.06 \%$ for ZRP with zone radius 4 , and $62.80 \%$ for ZRP with zone radius 5 . The reason is that with a small zone radius, the reactive routing mechanism (IERP) is mostly used. Therefore there is more than one RREP for each RREQ and ZRP keeps track of several routes between source and destination. In the case where one path is broken, an alternative path is chosen, and as a result, the TP increases. 


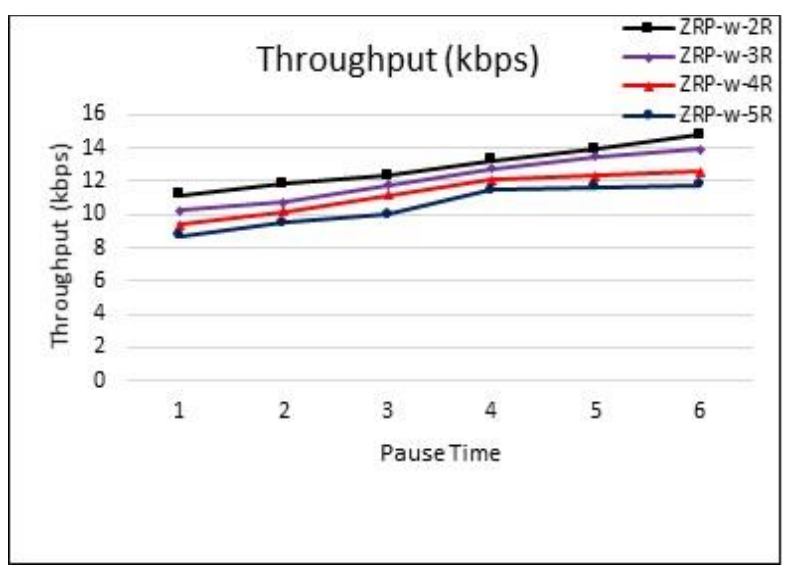

Figure 5. TP for scenario 1

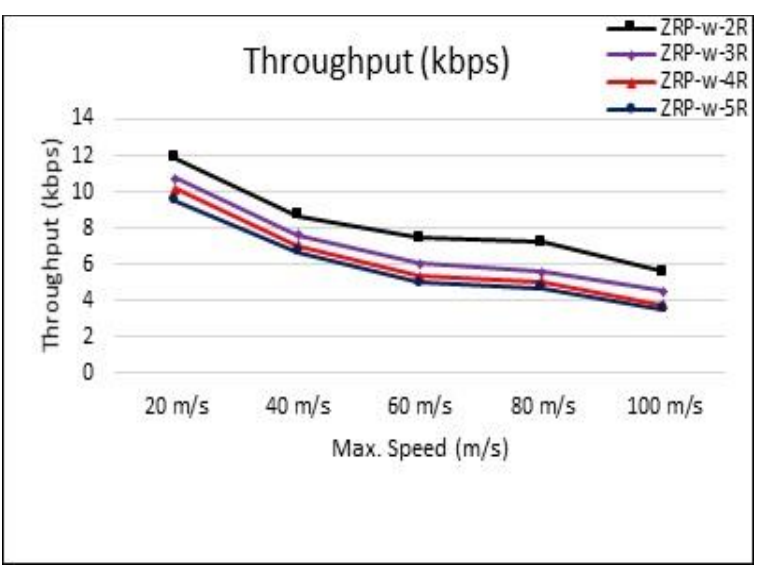

Figure 6. TP for scenario 2

\subsection{Results and analysis of NRL}

Figures 7 and 8 show the simulation results for ZRP in the two scenarios in terms of NRL. Figure 7 shows the NRL with respect to pause time. All four test cases show a decrease in NRL as the pause time increases. ZRP with zone radius 2 decreases from 50.772 to 30.372 , ZRP with zone radius 3 decreases from 67.766 to 39.573 , ZRP with zone radius 4 decreases from 91.725 to 53.978 , ZRP with zone radius 5 decreases from 127.162 to 68.04 when the pause time increases from 0 to 100 seconds. Low node movement leads to a stable network. Even though all the four test cases show a decrease in NRL as the pause time increases, but the decrease varies in each test case. The percentage of decrease in ZRP with zone radius 2 is $40.18 \%, 41.60 \%$ for ZRP with zone radius 3, 41.15\% for ZRP with zone radius 4, and 46.49\% for ZRP with zone radius 5. ZRP with zone radius 2 has a lower NRL compared to the other three test cases because, with a small number of zone radius, IERP is used to communicate with nodes outside the zone. IARP provides the routing information for IERP to direct query requests to the peripheral nodes using BRP and then uses a reactive mechanism to search for a path to the destination. In the case where several replies are received for the same route request, these are considered as redundant and are discarded by Early Termination (ET) based on advanced query detection and knowledge of the local topology. Hence, the NRL is reduced.

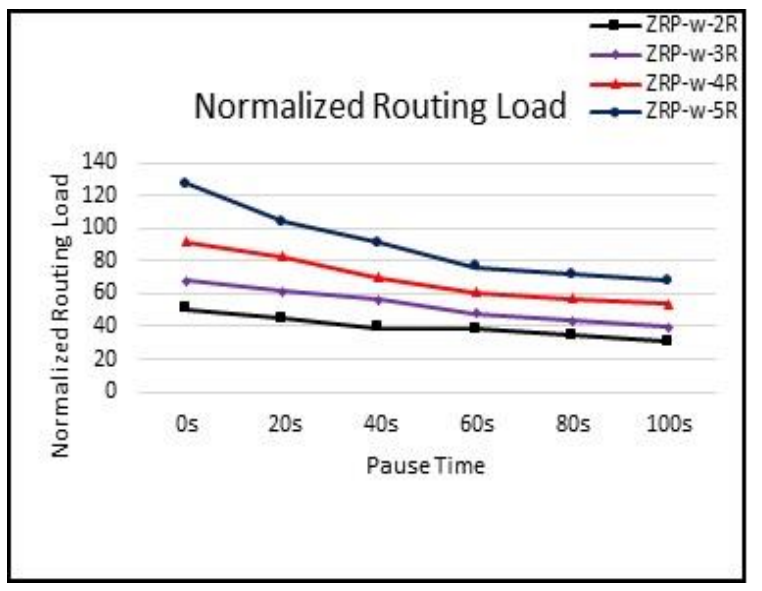

Figure 7. NRL for scenario 1

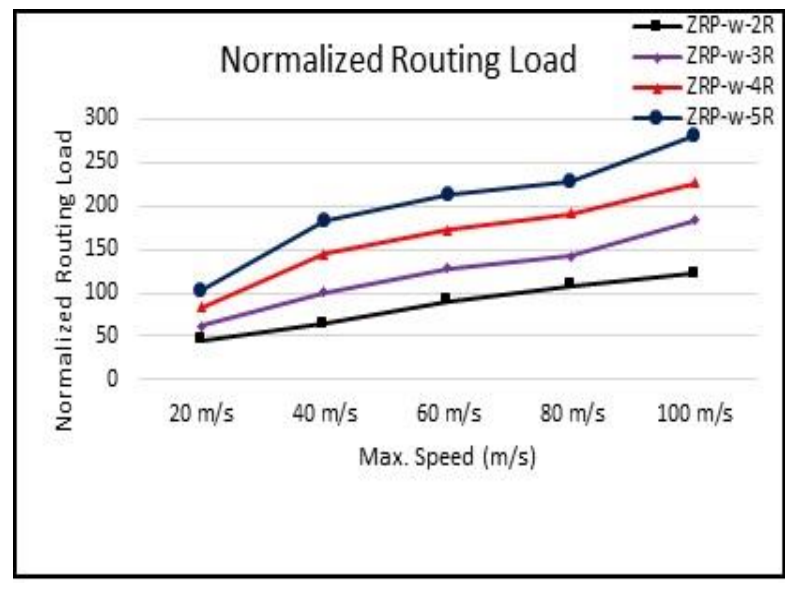

Figure 8. NRL for scenario 2

Figure 8 shows the NRL with respect to node speed. As expected, all four test cases show an increase in NRL when node speed increases. This is because high node speed usually leads to highly dynamic network topology and causes frequent path re-establishment. The NRL for ZRP with zone radius 2 increases from 45.044 to 121.721 , ZRP with zone radius 3 increases from 61.826 to 184.141 , ZRP with zone radius 4 increases from 82.757 to 225.796 , and ZRP with zone radius 5 increases from 103.668 to 279.92 . The percentage of increase in NRL for ZRP with zone radius 2 as compared to ZRP with zone radius 3, 4, and 5 
at the node speed $100 \mathrm{~m} / \mathrm{s}$ is about $170.22 \%, 197.84 \%, 172.84 \%$, and $170.02 \%$ respectively. ZRP with zone radius 2 has a lower NRL compared to the other three test cases. The reason is that, with a small zone radius, the IERP packets dominate a majority of the traffic. Therefore, when links are broken due to node movement, an alternative path can be chosen since there is more than one RREP for a single RREQ. As a result, NRL for ZRP with zone radius 2 is lower than ZRP with zone radius 3, 4, and 5.

\section{CONCLUSION}

The evaluation of ZRP performance has been done based on PDF, average E-to-E delay, TP, and NRL with respect to different levels of node mobility (i.e. node speed and pause time) and zone radius. With varying pause time, the results have shown that the performance of ZRP with zone radius 2, 3, 4, and 5 increases significantly in terms of PDF, TP, average E-to-E delay, and NRL. The reason is when the pause time increases, the movement of nodes decreases and the networks become more stable. As a result, the probability of transmission failure due to a broken link is lower. Whereas when increasing the maximum speed of the nodes, the performance of ZRP with zone radius 2, 3, 4, and 5 decreases as has appeared in three performance metrics are packet delivery fraction, throughput, and NRL. This is due to the topology of the network is changing dynamically because of the high speed of nodes. But the opposite happened with the average E-to-E delay has decreased due to since ZRP is a hybrid routing protocol, it provides a balance between overhead caused by proactive routing and latency caused by reactive routing. However, ZRP with zone radius 2 provides the best PDF, TP and NRL compared to ZRP with zone radius 3, 4, and 5 but with the cost of delay. This is because, with a small zone radius, most of the destination nodes lie outside the routing zone; therefore, a reactive routing mechanism is required to find the route to destinations. This mechanism requires a longer setup delay to discover the route, but with a negligible effect on the performance of ZRP. Therefore, zone radius 2 is the optimum zone radius for ZRP based on node mobility.

\section{REFERENCES}

[1] N. S. Saba Farheen and A. Jain, "Improved routing in MANET with optimized multi path routing fine tuned with hybrid modeling," J. King Saud Univ. - Comput. Inf. Sci., pp. 1-8, 2020, doi: 10.1016/j.jksuci.2020.01.001.

[2] S. Zafar, S. Khan, N. Iftekhar, and S. S. Biswas, "Substantiation of Vulnerabilities in Various Secure Routing Protocols of MANET," in Forensic Investigations and Risk Management in Mobile and Wireless Communications. IGI Global, pp. 168-194, 2020.

[3] M. H. Hassan, S. A. Mostafa, A. Budiyono, A. Mustapha, and S. S. Gunasekaran, "A hybrid algorithm for improving the quality of service in MANET," Int. J. Adv. Sci. Eng. Inf. Technol., vol. 8, no. 4, pp. 1218-1225, 2018, doi: 10.18517/ijaseit.8.4.5004.

[4] P. Khatri and P. R. Rajvanshi, "A Relative Study About Mobile Ad-Hoc Network (MANET): Applications, Standard, Protocols, Architecture, and Recent Trends," in IoT and Cloud Computing Advancements in Vehicular Ad-Hoc Networks. IGI Global, pp. 156-173, 2020.

[5] A. S. Mahmoud, A. Bassel, and H. M. Haglan, "Effect of Node Speed and Packet Size on thePerformance of the Routing Protocols in Mobile Ad-HocNetwork (MANET)," AUS Rev., vol. 26, no. 2, pp. 200-207, 2019, doi: 10.4206/aus.2019.n26.2.26/.

[6] N. Raza, M. U. Aftab, M. Q. Akbar, O. Ashraf, and M. Irfan, "Mobile Ad-Hoc Networks Applications and Its Challenges," Commun. Netw., vol. 8, no. 3, pp. 131-136, 2016.

[7] M. Rath and B. K. Pattanayak, "Monitoring of QoS in MANET based real time applications," in International Conference on Information and Communication Technology for Intelligent Systems. Springer, Cham, pp. 579-586, 2017.

[8] D. Zhang, J. Gao, X. Liu, T. Zhang, and D. Zhao, "Novel approach of distributed \& adaptive trust metrics for MANET," Wirel. Netw., vol. 25, no. 6, pp. 3587-3603, 2019.

[9] S. H. H. Nazhad, M. Shojafar, S. Shamshirband, and M. Conti, "An efficient routing protocol for the QoS support of large-scale MANETs,” Int. J. Commun. Syst., vol. 31, no. 1, pp. 1-14, 2018, doi: 10.1002/dac.3384.

[10] F. T. Al-Dhief, N. Sabri, M. S. Salim, S. Fouad, and S. A. Aljunid, "MANET Routing Protocols Evaluation: AODV, DSR and DSDV Perspective," in MATEC Web of Conferences, vol. 150, pp. 1-6, 2018, doi: 10.1051/matecconf/201815006024.

[11] P. Nayak and B. Vathasavai, "Impact of random mobility models for reactive routing protocols over MANET," Int. J. Simul. Syst. Sci. Technol., vol. 17, no. 34, pp. 112-115, 2016, doi: 10.5013/IJSSST.a.17.34.13.

[12] P. Jain and A. Sanghi, "Review of Various Routing Protocols in Mobile Ad-Hoc Networks (MANETs)," Akash Sanghi Int. J. Innov. Adv. Comput. Sci. IJIACS ISSN, vol. 7, no. 4, pp. 2347-8616, 2018, [Online]. Available: http://academicscience.co.in/admin/resources/project/paper/f201804091523290082.pdf.

[13] S. Liu et al., "Dynamic Analysis for the Average Shortest Path Length of Mobile Ad Hoc Networks under Random Failure Scenarios," IEEE Access, vol. 7, pp. 21343-21358, 2019, doi: 10.1109/ACCESS.2019.2896699.

[14] X. Fan, W. Cai, and J. Lin, "A survey of routing protocols for highly dynamic mobile ad hoc networks," in International Conference on Communication Technology Proceedings, ICCT, 2017-October, pp. 1412-1417, 2018, doi: 10.1109/ICCT.2017.8359865. 
[15] D. Zhang, T. Zhang, Y. Dong, X. Liu, Y. Cui, and De-xinZhao, "Novel optimized link state routing protocol based on quantum genetic strategy for mobile learning," J. Netw. Comput. Appl., vol. 122, pp. 37-49, 2018.

[16] J. Shen, C. Wang, A. Wang, X. Sun, S. Moh, and P. C. K. Hung, "Organized topology based routing protocol in incompletely predictable ad-hoc networks,” Comput. Commun., vol. 99, pp. 107-118, 2017, doi: 10.1016/j.comcom.2016.07.009.

[17] Y. Bai, Y. Mai, and N. Wang, "Performance comparison and evaluation of the proactive and reactive routing protocols for MANETs," in Wireless Telecommunications Symposium, 2017, pp. 1-5, doi: 10.1109/WTS.2017.7943538.

[18] H. M. Haglan et al., "Analyzing the impact of the number of nodes on the performance of the routing protocols in MANET environment," Bull. Electr. Eng. Informatics, vol. 10, no. 1, 2020.

[19] S. Shruthi, "Proactive routing protocols for a MANET-A review," in Proceedings of the International Conference on IoT in Social, Mobile, Analytics and Cloud, I-SMAC 2017, pp. 821-827, 2017, doi: 10.1109/ISMAC.2017.8058294.

[20] M. P. Manohar and S. P. Setty, "Performance Analysis of Reactive Routing Protocols AODV, DYMO, DSR, LAR in MANETs," Int. J. Futur. Revolut. Comput. Sci. Commun. Eng., vol. 4, no. 3, pp. 1-7, 2018.

[21] M. H. Hassan and R. C. Muniyandi, "An improved hybrid technique for energy and delay routing in mobile ad-hoc networks," Int. J. Appl. Eng. Res., vol. 12, no. 1, pp. 134-139, 2017.

[22] K. W. Al-Ani, S. Yussof, H. M. Haglan, and H. Shaker, "Review on Routing Protocols for Mobile Ad-Hoc Network," Eur. J. Sci. Res., vol. 117, no. 4, pp. 568-584, 2014.

[23] Y. Jahir, M. Atiquzzaman, H. Refai, A. Paranjothi, and P. G. LoPresti, "Routing protocols and architecture for Disaster Area Network: A survey," Ad Hoc Networks, vol. 82, pp. 1-14, 2019, doi: 10.1016/j.adhoc.2018.08.005.

[24] H. M. Haglan, S. Yussof, K. W. Al-Ani, H. S. Jassim, and D. A. Jasm, "The effect of network size and density to the choice of zone radius in ZRP," Indones. J. Electr. Eng. Comput. Sci. (IJEECS), vol. 20, no. 1, pp. 206-213, 2020, doi: 10.11591/ijeecs.v20.i1.pp206-213.

[25] M. A. Jubair et al., "Bat optimized link state routing protocol for energy-aware mobile ad-hoc networks," Symmetry (Basel)., vol. 11, no. 11, pp. 1-22, 2019, doi: 10.3390/sym11111409.

[26] A. O. A. Salem, G. Samara, and T. Alhmiedat, "Performance Analysis of Dynamic Source Routing Protocol," J. Emerg. Trends Comput. Inf. Sci., vol. 5, no. 2, pp. 97-100, 2017.

[27] D. J. Persis and T. P. Robert, "Review of ad-hoc on-demand distance vector protocol and its swarm intelligent variants for mobile ad-hoc network," IET Networks, vol. 6, no. 5, pp. 87-93, 2017, doi: 10.1049/iet-net.2017.0015.

[28] A. S. Toor and A. K. Jain, "A survey on wireless network simulators," Bull. Electr. Eng. Informatics, vol. 6, no. 1, pp. 62-69, 2017, doi: 10.11591/eei.v6i1.568.

[29] R. Raman Chandan, B. Singh Kushwaha, and P. Kumar Mishra, "Performance Evaluation of AODV, DSDV, OLSR Routing Protocols using NS-3 Simulator,” Int. J. Comput. Netw. Inf. Secur., vol. 10, no. 7, pp. 59-65, 2018, doi: 10.5815/ijcnis.2018.07.07.

[30] N. Muthukumaran, "Analyzing Throughput of MANET with Reduced Packet Loss," Wirel. Pers. Commun., vol. 97, no. 1, pp. 565-578, 2017, doi: 10.1007/s11277-017-4520-9.

\section{BIOGRAPHIES OF AUTHORS}

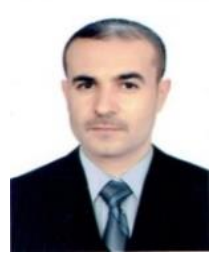

Khalid Waleed Kadhim was born in Al-Anbar Governorate, Iraq in 1982. He received the B.Sc. Computer Engineering and Information Technology degree from the University of Technology, Iraq in 2005 and the Master degree in Information Technology from University Tenaga Nasional (UNITEN), Malaysia in 2014. He is currently one of the teaching staff at the University of Anbar, Iraq. His research interests include data communication and networks, big data, data base, and artificial intelligence.

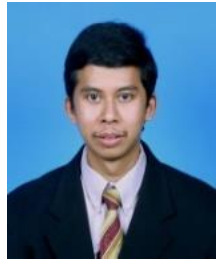

Salman Yussof is an Associate Professor at the College of Computing and Informatics, Universiti Tenaga Nasional, Malaysia. He received his Bachelor of Science degree and Masters of Science degree in Electrical and Computer Engineering from Carnegie Mellon University, USA, in 1999. In the same year, he was accepted as a faculty member at Universiti Tenaga Nasional. While working as a faculty member, he pursued his PhD study in the same university and eventually received his PhD in 2010 . His research interests include next generation Internet technologies, network security and security for critical infrastructure. He is a member of IEEE.

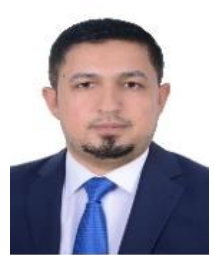

Hussein Mahdi Haglan was born in Al-Anbar Province, Iraq in 1984. He received the B.Sc. degree in Information System from the University of Anbar, Ramadi, Iraq in 2006 and the Master degree in Information Technology from University Tenaga Nasional (UNITEN), Malaysia in 2014. He is currently one of the teaching staff at the University of Anbar, Iraq. His research interests include data communication and networks, data mining, big data, data science, and artificial intelligence. 